\title{
Transient Expression of c-Fos in Rat Amygdala During Training Is Required for Encoding Conditioned Taste Aversion Memory
}

\author{
Raphael Lamprecht and Yadin Dudai ${ }^{1}$ \\ Department of Neurobiology \\ The Weizmann Institute of Science \\ Rehovot 76100 , Israel
}

\begin{abstract}
Local microinjection into rat amygdala of phosphorothioate modified oligodeoxynucleotides (ODNs) antisense to c-fos several hours before conditioned taste aversion (CTA) training impaired taste aversion memory tested 3-5 days after conditioning. In contrast, injection of the antisense ODNs several days before training, before testing, or into the basal ganglia, or injection of c-fos sense ODNs, had no effect on CTA memory. Inhibition of translation by local microinjection of anisomycin into the amygdala shortly before as well as during CTA training, but not several days before training or shortly before testing, also impaired CTA memory. We conclude that translation in general, and c-Fos translation in particular, in the amygdala during or immediately after CTA training is essential for encoding taste aversion memory.
\end{abstract}

\section{Introduction}

In conditioned taste aversion (CTA), organisms learn to avoid a taste if the first encounter with that taste is followed, even many hours later, by reversible poisoning (Revusky and Garcia 1970). The fast learning (single trial), robust memory (weeks to a lifetime), and delayed associability of the conditioned and unconditioned stimuli turn CTA into a useful system for analyzing molecular,

\footnotetext{
${ }^{1}$ Corresponding author.
}

cellular, and neuroanatomical mechanisms of acquisition, association, consolidation, retention, and retrieval of discrete items in memory. The neural mechanisms that subserve the representation in the brain of taste and malaise, their association in CTA, and the retention of that association over time have not yet been elucidated, but several brain areas already have been implicated in these processes (for review, see Bures et al. 1988; Yamamoto et al. 1994). Thus, it is generally accepted that the gustatory cortex (GC) in the insular cortex plays a role in the initial processing of the taste representation, the parabrachial nucleus (PBN) in the association between taste and malaise, and the amygdala in the integration and the expression of CTA behavior.

Within each of the above and other relevant brain structures, one should expect to identify molecular processes that subserve and embody the neuronal representations of the learned behavior (Dudai 1989). We have reported that in the insular cortex, CTA training is correlated with enhanced protein tyrosine phosphorylation of synaptic proteins (Rosenblum et al. 1995), and that in the same cortex the encoding of memory for the novel taste requires intact cholinergic function (Naor and Dudai 1996) and protein synthesis (Rosenblum et al. 1993). Moreover, by use of in situ hybridization we have found recently that administration of the malaise-inducing agent commonly employed in CTA training, $\mathrm{LiCl}$ i.p., is associated with differential and combinatorial modulation of the mRNA of several immediate-early genes (IEGs), including c-fos, in the nucleus of the solitary tract (NTS), parabrachial nucleus (PBN), hypothalamic paraventricular nucleus (PVN), and central amygdaloid nucleus (CeA) (Lamprecht and Dudai 1995), areas that have been implicated

LEARNING \& MEMORY 3:31-41 (c) 1996 by Cold Spring Harbor Laboratory Press ISSN1072-0502/96 \$5.00

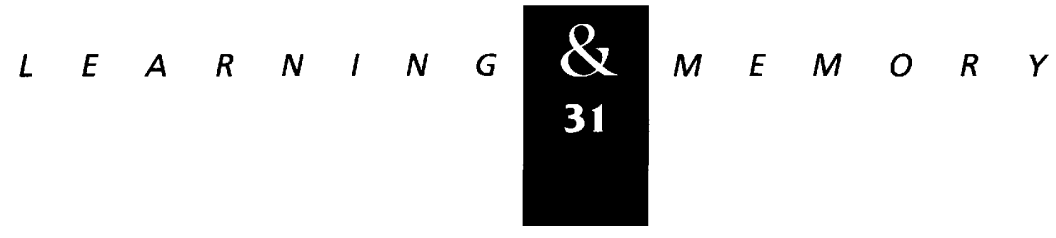


previously in CTA by other studies (Yamamoto et al. 1994).

Observations that the formation of taste memory depends on brain protein synthesis, and that the administration of the malaise-inducing agent correlates with modulation of the expression of IEGs in the brain, are in consonance with the prevailing molecular hypothesis that the encoding of long-term memory traces involves modulation of neuronal gene expression (Davis and Squire 1984; Goelet et al. 1986; Dudai 1989). Within this molecular framework, inhibition of protein synthesis during a sensitive time window overlapping or immediately following training should disrupt the expression of stimulus-induced IEG products; these normally regulate the expression of late response genes, culminating in enduring structural and functional alterations in the circuit that encodes the stored representation. Guided by the aforementioned hypothesis, we tested whether the expression of an IEG in a brain region implicated in CTA by multiple experimental approaches is, indeed, obligatory for the encoding of CTA memory or, alternatively, is merely correlated with CTA training.

We have chosen to concentrate on the expression of c-fos in the amygdala. Evidence indicating a role for the amygdala in CTA is based on a multiplicity of lesion techniques, including permanent (anatomical) and transient (metabolic) lesions inflicted at various time points before, during, and after CTA training (Gallo et al. 1992; Roldan and Bures 1994; Yamamoto et al. 1994). It is also supported by the neuroanatomical links of the amygdala to other brain areas implicated in CTA, including the NTS, PBN, thalamus, and insular cortex (Norgren 1976; van der Kooy et al. 1984; Turner and Hrekenham 1991), and by the role assigned to the amygdala in other types of learning, especially aversive and emotional conditioning (Davis 1992; LeDoux 1993; McGaugh et al. 1993; Cahill et al. 1995). Furthermore, of the IEGs' mRNA we tested, the modulation of c-fos in the amygdala in response to $\mathrm{LiCl}$ i.p. was one of the most prominent (Lamprecht and Dudai 1995). An increase in c-Fos immunoreactivity in the amygdala following $\mathrm{LiCl}$ injection i.p. was reported by Gu et al. (1992) and Yamamoto et al. (1993).

A potentially effective methodology to probe the functional role in specific behaviors of gene products in circumscribed brain regions is to selectively, locally, and transiently block the expression of these gene products by microinjection of antisense oligodeoxynucleotides (ODNs) (Chiasson et al. 1994; Wahlestedt 1994). The antisense ODNs are expected to bind to the target mRNA and, hence, specifically block translation (Helene 1991 ). This methodology has been used successfully to block the expression of several genes in the brain, including c-fos, and to modulate certain behaviors (Dragunow et al. 1993; Heilig et al. 1993; Sommer et al. 1993; Moller et al. 1994; Cirelli et al. 1995; Landgraf et al. 1995). We have used this approach to analyze the role in CTA of c-Fos in the amygdala. In a complementary set of experiments, we have transiently inhibited translation in the amygdala by local microinjection of anisomycin during the time window in which c-Fos is expressed in CTA training (Lamprecht and Dudai 1995). Our data indicate that in the amygdala, translation in general, and c-Fos translation in particular, during or immediately after CTA training is required for encoding normal CTA memory.

\section{Materials and Methods}

\section{ANIMALS}

Male Wistar rats ( 2 months old, 200-250 g) were caged individually at $22^{\circ} \mathrm{C}$ in $12: 12 \mathrm{hr}$ light/ dark cycles. Water and food were available ad libitum unless otherwise indicated.

\section{REAGENTS}

The phosphorothioate-modified ODNs were synthesized at the Biological Services of the Weizmann Institute of Science. The sequence for c-fos antisense was 5'-GAACATCATGGTCGT-3' and for c-fos sense was 5'-ACGACCATGATGTTC-3'. ODNs were injected in physiological saline $(0.9 \%$ $\mathrm{NaCl}$ ). Anti-Fos polyclonal antibody (SC-25) was from Santa Cruz. Biotinylated goat anti-rabbit antibody (BA 1000) and avidin DH-biotinylated horseradish peroxidase $\mathrm{H}$ complex (PK-6100) were from Vector. Anisomycin (A9789), 3',3'-diaminobenzidine tetrahydrochloride (DAB) tablets (D-4293), cytochrome C (C-7752), and catalase (C-40) were from Sigma. All other chemicals were of analytical grade or the highest grade available.

\section{CTA TRAINING AND TESTING}

Saccharin $(0.1 \%$, sodium salt $)$ was used as an unfamiliar taste, and $\mathrm{LiCl}$ i.p. $(0.15 \mathrm{~m}, 2 \%$ body

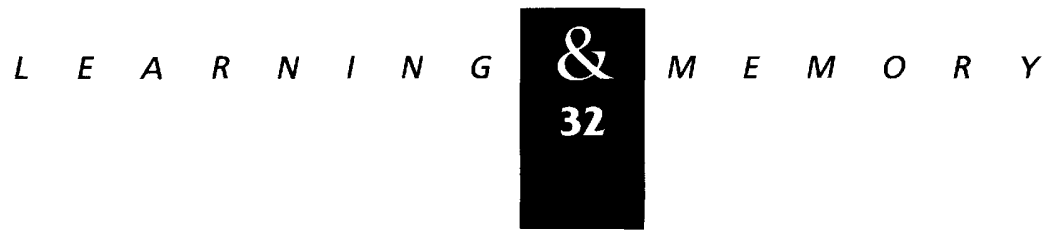


weight) was used as the malaise-inducing agent. The CTA protocol was as described by Rosenblum et al. ( 1993). In brief, rats were trained over 4 days to get their daily water ration within $10 \mathrm{~min}$ from 2 pipettes, each containing $10 \mathrm{ml}$. On day 5 (conditioning day), the rats were presented with saccharin instead of water. Forty minutes later, they were injected with $\mathrm{LiCl}$. On days $6-7$ or $6-8$, the rats were again presented with water. Testing was performed in an extinction mode on days 8-10 or 9-11; the rats were presented daily for $15 \mathrm{~min}$ with an array of 6 pipettes, three containing $4 \mathrm{ml}$ saccharin and three containing $4 \mathrm{ml}$ water, and their liquid consumption was recorded. In one of the anisomycin experiments, indications were obtained for a reduction in the total amount of drinking by the experimental animals, and in that experiment the drinking period was extended to $\mathbf{5 0}$ min to ensure that all the groups received ample opportunity to consume the liquid. The aversion index (Rosenblum et al. 1993) was defined as $\{[\mathrm{ml}$ water $/(\mathrm{ml}$ water $+\mathrm{ml}$ saccharin $)] \times 100\}$ consumed in the test, that is, 50 is chance level, and the higher the aversive index, the more the rats prefer water to saccharin.

\section{SURGICAL PROCEDURES}

Microinjection into the amygdala was performed via chronically implanted cannulae. For surgery, rats were anesthetized with Equithesin $(5.5 \mathrm{ml} / \mathrm{kg})$, restrained in a stereotactic apparatus (Kopf), and implanted bilateraly with guide cannulae (stainless steel, 23 gauge) aimed at the central amygdaloid nucleus (coordinates in reference to Bregma AP - 2.3, L + 4, DV - 7.5; Paxinos and Watson 1986). In some control experiments, the guide cannula was implanted in the basal ganglia (coordinates in reference to Bregma AP $-2.3, \mathrm{~L}$ +4 , DV -5.5). The cannulae were fixed in place with acrylic dental cement and secured by two skull screws. A stylus was placed in the guide cannula to prevent clogging. Animals were allowed 1 week to recuperate before being subjected to experimental manipulations.

\section{MICROINJECTION}

Injection of ODNs ( 5 nmole in $2 \mu \mathrm{l} / \mathrm{hemi-}$ sphere) was performed at the times indicated in the Results. The stylus was removed from the guide cannula and a 28-gauge injection cannula was carefully inserted. The injection cannula was connected via PE20 tubing to a Hamilton microsyringe, driven by a microinjection pump (Carnegie Medicine CMA 100) at a rate of $0.5 \mu \mathrm{l} / \mathrm{min}$. Following injection, the injection cannula was left for an additional $1 \mathrm{~min}$ before withdrawal to reduce dragging of injected liquid along the injection track.

In four rats, India ink ( $2 \mu \mathrm{l} /$ hemisphere ) was injected bilaterally into the amygdala at the aforementioned coordinates to estimate the sphere of diffusion of the injected solutions (Fig. 1A). We estimated that the solution diffused into a maximal area of $2.1 \pm 0.4 \mathrm{~mm}^{2}$, centered around the CeA. In a few sections, the microinjected solution extended into one or more of the following structures: dorsally into the ventral horn of the caudate putamen, dorsomedially into the nucleus basalis of Meynert and the dorsal portion of the internal capsule, and ventrolaterally into the intercalated amygdaloid nuclei, the amygdaloid intermedullary gray, and the basolateral amygdala. The only common feature of the injections, however, was the bilateral involvement of substantial portions of the CeA.

\section{HISTOCHEMISTRY}

For immunohistochemical determination of c-Fos, rats were anesthetized with Equithesin (5.5 $\mathrm{ml} / \mathrm{kg}$ ) $90 \mathrm{~min}$ after administration of LiCl in CTA training and perfused intracardially by gravity-drip infusion with phosphate-buffered saline (PBS) followed by cold $\left(4^{\circ} \mathrm{C}\right) 2.5 \%$ paraformaldehyde, $5 \%$ sucrose in PBS at $\mathrm{pH} 7.4$. Brains were postfixed for $48 \mathrm{hr}$ in $1 \%$ paraformaldehyde, $30 \%$ sucrose in PBS at $4^{\circ} \mathrm{C}$.

Postfixed brains were sectioned coronally on a freezing microtome at a thickness of $50 \mu \mathrm{m}$. Sections were washed three times ( 5 min each) with PBS and immersed for $30 \mathrm{~min}$ in $49.5 \%$ methanol, $49.5 \% \mathrm{PBS}$, and $1 \% \mathrm{H}_{2} \mathrm{O}_{2}$, followed by five washings ( 5 min each) with PBS and immersion for 20 $\mathrm{min}$ in $0.15 \mathrm{M}$ glycine in PBS at $\mathrm{pH} 7.4$. The sections were then blocked with $20 \%$ normal goat serum (NGS) in PBS and $0.5 \%$ Triton X-100 for 3 $\mathrm{hr}$ at $37^{\circ} \mathrm{C}$, followed by $12 \mathrm{hr}$ incubation at room temperature with the c-Fos polyclonal antibody (1:500) and $2 \%$ NGS in PBS. The sections were washed three times ( $5 \mathrm{~min}$ each) with PBS and incubated with biotinylated goat anti-rabbit anti-

$$
\text { ….... }
$$


Figure 1: The localization of microinjections. (A) Camera lucida drawings of serial sections (1-3) depicting the area of diffusion of India ink, microinjected under the conditions detailed in Materials and Methods, in a representative rat (brain sections adapted from the atlas of Paxinos and Watson 1986). (B) Photomicrographs of sections stained for $\mathrm{cy}$ tochrome oxidase activity (1-3) and Nissl (4-6) from rats microinjected with saline $(1,4)$, ODN antisense to c-fos $(2,5)$, and anisomycin $(3,6)$. In each pair, successive sections are shown. For further details and quantification, see text.


body (1:200) and 2\% NGS in PBS for $1.5 \mathrm{hr}$ at room temperature followed by three additional PBS washings ( 5 min each). Finally, an avidin DHbiotinylated horseradish peroxidase $\mathrm{H}$ complex (1:25) was added for $30 \mathrm{~min}$. The sections were then washed once with PBS and twice with $50 \mathrm{~mm}$ Tris- $\mathrm{Cl}$ buffer ( $\mathrm{pH} 7.3$ ). Peroxidase activity was determined by reaction with a mixture containing $0.7 \mathrm{mg} / \mathrm{ml}$ of DAB, $0.7 \mathrm{mg} / \mathrm{ml}$ of urea- $\mathrm{H}_{2} \mathrm{O}_{2}$ in 60 mM Tris $-\mathrm{Cl}$. The sections were washed three times with Tris buffer, mounted on slides, dehydrated by successive rinses of $70 \%, 80 \%, 95 \%$, and $100 \%$ ethanol $(\times 2)$ and $100 \%$ xylene $(\times 2)$, and covered with Permount.

For cytochrome oxidase staining, rats were anesthetized as above 4 days after the microinjec- tion of ODNs, saline, or anisomycin into the amygdala, and perfused intracardially by gravitydrip infusion with PBS followed by cold $\left(4^{\circ} \mathrm{C}\right)$ $2.5 \%$ glutaraldehyde, $0.5 \%$ paraformaldehyde, and $5 \%$ sucrose in PBS. Brains were postfixed for 12 hrs in $2.5 \%$ glutaraldehyde, $0.5 \%$ paraformaldehyde, and $15 \%$ sucrose in PBS at $4^{\circ} \mathrm{C}$. Postfixed brains were sectioned coronally on a freezing $\mathrm{mi}$ crotome at $50 \mu \mathrm{m}$, washed four times, $5 \mathrm{~min}$ each, in phosphate buffer ( $\mathrm{pH} 7.6)$ and incubated for 3 hrs at $40^{\circ} \mathrm{C}$ in an oxygenated reaction mixture containing $10 \mathrm{mg}$ of DAB, $1.5 \mathrm{mg}$ of cytochrome c, and $1.6 \mathrm{mg}$ of catalase (in $0.1 \%$ thymol) in phosphate buffer ( $\mathrm{pH}$ 7.6). The sections were then mounted on slides and dehydrated by successive rinses in $50 \%, 70 \%, 95 \%$, and $100 \%$ ethanol $(\times 2)$

$$
\text { (........ }
$$


and $100 \%$ xylene $(\times 2)$, and covered with Permount.

For Nissl staining, brains were processed as above. Sections were mounted on slides and dehydrated by successive rinses in $70 \%, 90 \%, 95 \%$, and $100 \%(\times 2)$ ethanol followed by $30 \mathrm{~min}$ in chloroform. The slides were then rehydrated by successive rinses in $100 \%, 95 \%, 90 \%$, and $70 \%$ ethanol and double-distilled water (DDW). The slides were then stained with $0.5 \%$ cresyl violet, bleached with $50 \%$ ethanol and $1 \%$ acetic acid, rinsed in $70 \%(\times 3), 90 \%, 95 \%$, and $100 \%(\times 2)$ ethanol and $100 \%$ xylene $(\mathrm{x} 2)$, and covered with Permount.

A Nikon Labophot microscope and Image 1.41 software (NIH) were used for histochemical analyses, except that Nissl stained cells were quantified manually under the microscope using a gridded eye lens at a magnification of $\times 200$.

\section{STATISTICS}

Differences among groups for the behavioral and immunohistochemical results were evaluated by use of one-way ANOVA, and for repeated measure tests, a two-way ANOVA. For paired comparisons, Scheffe contrast tests were used with an $\alpha$ level of 0.05 .

\section{Results}

MICROINJECTION OF c-fos ANTISENSE INTO THE AMYGDALA SHORTLY BEFORE TRAINING IMPAIRED CTA MEMORY

Previous reports have shown that the effect of phosphorothioate-modified ODNs is obtained optimally when they are microinjected into the brain 8-10 hours before expression of the physiological or behavioral variable they are supposed to affect (Heilig et al. 1993; Chiasson et al. 1994; Moller et al. 1994). Therefore, we microinjected the ODNs into the amygdala $8 \mathrm{hr}$ before CTA training. As can be seen in Fig. 2, c-fos antisense in the amygdala markedly reduced CTA memory, whereas c-fos sense had no effect. Differences among groups were significant for the first two postconditioning test days $(P<0.0002$ and $P<0.003$, respectively $)$. The antisense group showed the lowest aversion scores on all the tests. Their aversion index was significantly lower than that of the normal CTA controls and the sense group on the first test day,



Figure 2: Impairment of CTA by ODNs antisense to c-fos in the amygdala. Aversion indices are plotted vs. the test day. In each test day, the left (shaded) bar depicts the aversion index of normal CTA control animals $(n=12)$, the center (solid) bar of animals locally microinjected into the amygdala with antisense ODNs $(n=11)$, and the right (open) bar of animals receiving sense ODNs $(n=9)$. All microinjections into the amygdala were performed $8 \mathrm{hr}$ before CTA training, as detailed in Materials and Methods. (Inset) Spatial and temporal specificity of the antisense effect. As above, shaded bars depict the results for normal controls and solid bars for rats microinjected with antisense. (A) Antisense microinjected $2 \mathrm{~mm}$ above the stereotactic coordinates used for injection into the amygdala; $(B)$ antisense microinjected into the amygdala 4 days before CTA training; (C) antisense microinjected into the amygdala $8 \mathrm{hr}$ before the first memory test, i.e., $64 \mathrm{hr}$ after completion of training; $n=6$ animals in each group.

and significantly lower than that of the normal CTA controls on the second test day (Scheffe contrast tests). A group-by-days ANOVA demonstrated a significant difference among groups $(P<0.001)$ and a significant decrease in the aversion index over days $(P<0.005)$ with no interaction effect.

\section{MICROINJECTION OF c-fos ANTISENSE SEVERAL DAYS BEFORE TRAINING HAD NO EFFECT ON CTA MEMORY}

In contrast to its effect when microinjected hours before training, c-fos antisense had no effect on CTA memory when microinjected into the amygdala 4 days before training (Fig. 2, inset). The ODNs are expected to degrade within such a time interval (Chiasson et al. 1992). Thus, these results indicate that microinjection of ODNs into the

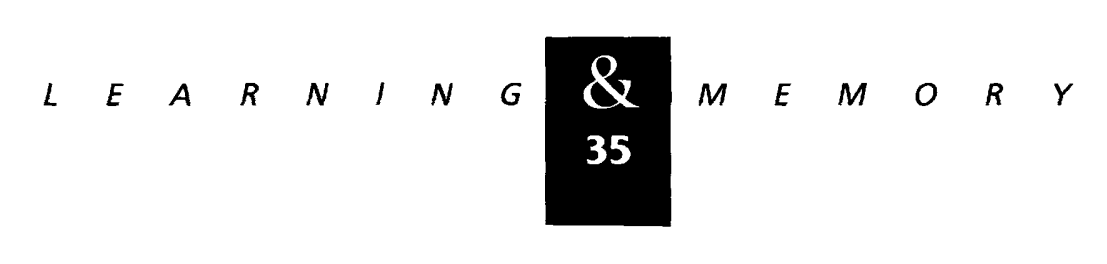


amygdala caused no residual functional damage to this structure with regard to CTA learning.

Furthermore, since ODNs may cause toxic effects, including structural damage in vivo (Chiasson et al. 1994), we have further tested the longterm effect of microinjection of c-fos antisense on the amygdala. This was done by comparing cytochrome oxidase- and Nissl-stained sections from animals microinjected into the amygdala with ODNs or saline $8 \mathrm{hr}$ before CTA training and sacrificed 4 days later. The small amount of tissue damage at the tip of the microinjection cannula did not differ among groups (maximal lesion area $0.6 \pm 0.2 \mathrm{~mm}^{2}$; Fig. 1B). In addition, the number of Nissl-stained cell bodies and the intensity of cytochrome oxidase activity were compared in selected frames below the injection cannula tip and found not to differ between antisense (A) and saline (S) groups (Nissl staining, $46 \pm 2$ (A) vs. $51 \pm 8$ (S) nuclei in $0.01 \mathrm{~mm}^{2}$ frames; cytochrome oxidase, $93 \pm 6$ (A) vs. $87 \pm 2$ (S), arbitrary pixel units in $0.4 \mathrm{~mm}^{2}$ frames ). Thus, we concluded that under the experimental conditions used here, the microinjection of the ODNs into the amygdala did not produce lasting tissue damage.

\section{MICROINJECTION OF c-fos ANTISENSE AFTER TRAINING AND SHORTLY BEFORE TESTING HAD NO EFFECT ON CTA MEMORY}

We tested whether c-fos antisense can also inhibit CTA if it is injected into the amygdala only after training is completed. We microinjected the ODNs into the amygdala $64 \mathrm{hr}$ after training, that is, $8 \mathrm{hr}$ before the first memory test (see the training and testing protocol in Materials and Methods). Under these conditions, the antisense had no effect on CTA memory (Fig. 2, inset). This also implies that microinjection of c-fos antisense into the amygdala had no effect on the detection of saccharin, on its conditioned hedonic valence, or on other sensory or motor faculties involved in the acquired rejection of the saccharin solution.

\section{MICROINJECTION OF c-fos ANTISENSE ABOVE THE AMYGDALA HAD NO EFFECT ON CTA MEMORY}

We also microinjected the ODNs into the basal ganglia instead of the amygdala $8 \mathrm{hr}$ before CTA training to verify that the effect is site-spe- cific. Indeed, such treatment had no effect on CTA memory (Fig. 2, inset).

MICROINJECTION OF c-fos ANTISENSE INTO THE AMYGDALA REDUCED THE LEVEL OF THE c-fos PROTEIN

The level of c-Fos protein in the amygdala was determined by immunohistochemistry. As reported earlier ( $\mathrm{Gu}$ et al. 1992; Yamamoto et al. 1993; Lamprecht and Dudai 1995), LiCl i.p. markedly increased the expression of c-Fos (Fig. 3). The differences among the saline/ $\mathrm{LiCl}$, sense/LiCl, and antisense/LiCl groups (Fig. 3, groups A-C) for density of c-Fos positive nuclei in $0.4 \mathrm{~mm}^{2}$ frames below the tip of the microinjection cannula were significant $(P<0.04)$. The antisense/LiCl group had substantially lower scores $(P<0.01)$ than the combined saline/ $\mathrm{LiCl}$ and sense/LiCl groups. The latter groups were combined for this comparison because there were only three rats in the saline/ $\mathrm{LiCl}$ group and their scores were essentially identical to the sense/LiCl group. In the absence of $\mathrm{LiCl}$

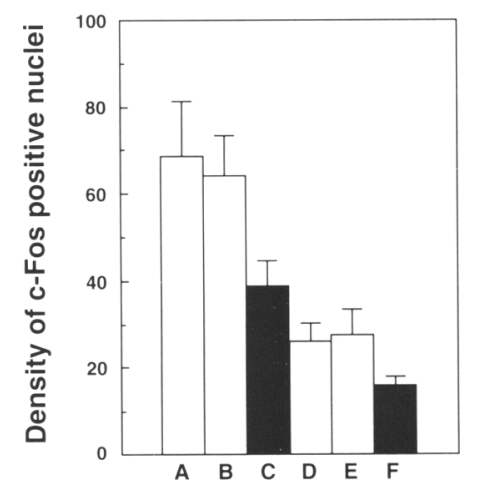

Figure 3: Inhibition of the stimulus-induced increase in the expression of c-Fos in the amygdala by microinjection of antisense to c-fos. Data are presented as density of c-Fos positive nuclei (number of nuclei in a 0.4 $\mathrm{mm}^{2}$ frame) vs. experimental group. In all the experimental groups, local microinjection into the amygdala was followed $8 \mathrm{hr}$ later by i.p. injection of $\mathrm{LiCl}$ or saline i.p. (A) Saline in the amygdala followed by $\mathrm{LiCl}$ i.p. $(n=3) ;(B)$ c-fos sense in the amygdala followed by $\mathrm{LiCl}$ i.p. $(n=7)$; (C) c-fos antisense in the amygdala followed by $\mathrm{LiCl}$ i.p. $(n=8) ;(D)$ saline in the amygdala followed by saline i.p. $(n=2)$; $(E)$ c-fos sense in the amygdala followed by saline i.p. $(n=3) ;(F)$ c-fos antisense in the amygdala followed by saline i.p. $(n=3)$. Combined data from two independent experiments, one containing groups $B$ and $C$ only and the other containing all the groups.

$$
\text { n....... }
$$


i.p., there was a trend for the antisense treatment to lower the level of c-Fos compared with the saline and sense groups (Fig. 3, groups D-F), although the differences among the groups under these low-expression conditions did not reach statistical significance.

\section{MICROINJECTION OF ANISOMYCIN INTO THE AMYGDALA-IMPAIRED CTA MEMORY}

Because c-fos antisense presumably inhibits c-Fos translation, one should also expect an impairment of CTA memory by other agents that inhibit IEGs' translation. A potent and relatively specific translational inhibitor is anisomycin, a blocker of the peptidyl transferase reaction (Grollman 1967). Anisomycin has been used successfully to block memory consolidation in several behavioral paradigms (Davis and Squire 1984), including the blockade of CTA memory by local microinjection into the rat insular cortex (Rosenblum et al. 1993). The effect of this antibiotic on memory consolidation is, indeed, commonly attributed to the translational arrest of IEGs (Goelet et al. 1986; Dudai 1989), although the actual effect of anisomycin and related compounds is rather complex and also involves transcriptional superinduction of IEGs and false nuclear signaling (Edwards and Mahadevan 1992).

We microinjected anisomycin locally and bilaterally into the amygdala, at the coordinates specified in Materials and Methods, $30 \mathrm{~min}$ before exposure to the unfamiliar taste in CTA training (Group $\mathrm{T}$ ) or $35 \mathrm{~min}$ before and again $30 \mathrm{~min}$ after the injection of $\mathrm{LiCl}$ i.p. (Group L). These time points were selected because our previous data indicate that anisomycin inhibits $>90 \%$ of protein synthesis in brain in vivo within $20 \mathrm{~min}$ of its introduction, and the inhibition lasts $>90 \mathrm{~min}$ (Rosenblum et al. 1993). Under these conditions, anisomycin significantly impaired CTA memory when tested a few days later (Fig. 4). Differences among groups were significant on each test day ( $P<0.0001, P<0.002$, and $P<0.005$ for days 1 to 3 , respectively), and on each test the group receiving anisomycin prior to exposure to the CS (Group T) showed the least aversion. This group had significantly lower aversion scores than control and Group $L$ rats on day 1 and significantly lower scores than the control on days 2 and 3 . In addition, the aversion index for Group $L$ was significantly lower than the control group on day 1

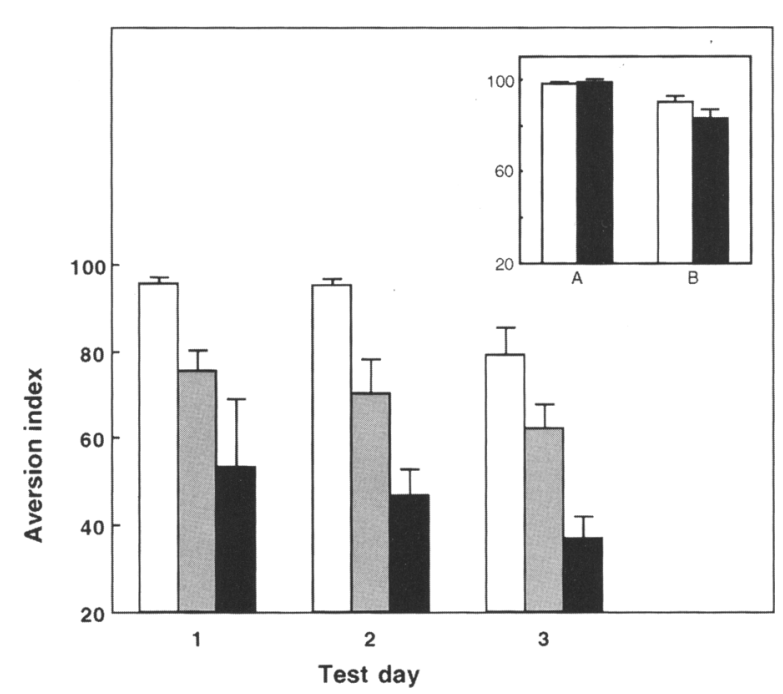

Figure 4: Impairment of CTA by anisomycin in the amygdala $(A)$ Aversion indices are plotted vs. the test day. In each test day, the left (open) bar depicts the CTA data for control animals ( $n=12$ ), the center (shaded) bar for animals locally microinjected into the amygdala with anisomycin $35 \mathrm{~min}$ before and $30 \mathrm{~min}$ after $\mathrm{LiCl}$ injection i.p. (i.e., the UCS in CTA training; $n=14$ ), and the right (solid) bar for animals microinjected into the amygdala with anisomycin $30 \mathrm{~min}$ before saccharin administration (i.e., CS) in CTA training $(n=5)$. (Inset) Temporal specificity of the anisomycin effect. (A) Anisomycin microinjected 4 days before CTA training; $(B)$ anisomycin microinjected into the amygdala $30 \mathrm{~min}$ before the first memory test, i.e., $72 \mathrm{hr}$ after training; $n=6$ animals in each group.

(Scheffe contrast tests). A group-by-days ANOVA demonstrated a significant difference among groups $(P<0.0001)$ and a significant decrease in the aversion index over days $(P<0.005)$ and no interaction effect.

Microinjection of anisomycin 4 days before training or 3 days after training and $30 \mathrm{~min}$ before the test had no effect (Fig. 4, inset), indicating that the microinjection of anisomycin had no long-lasting functional effect on the amygdala, nor did it impair the detection of the taste or its conditional hedonic valence. We also tested whether local microinjection of anisomycin into the amygdala resulted in tissue damage. This was done by comparing cytochrome oxidase and Nissl-stained sections from animals microinjected into the amygdala with anisomycin or saline $30 \mathrm{~min}$ before CTA training and sacrificed 3 days later (Fig. 1B). There was no evidence for anismoycin-specific tissue toxicity. In addition, the number of Nissl-stained cell bodies and the intensity of cytochrome oxi-

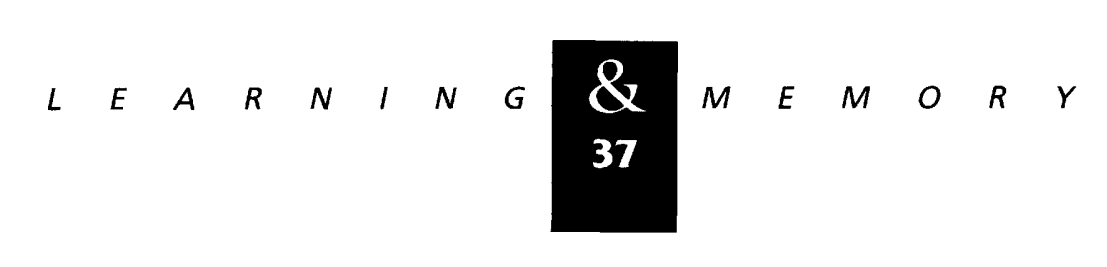


dase activity were compared in selected frames below the injection cannula tip and found not to differ between anisomycin (An) and saline (S) groups (Nissl staining, $47 \pm 5$ (An) vs. $51 \pm 8$ (S) nuclei in $0.01 \mathrm{~mm}^{2}$ frames; cytochrome oxidase, $90 \pm 16$ (An) vs. $87 \pm 2(\mathrm{~S})$, arbitrary pixel units in $0.4 \mathrm{~mm}^{2}$ frames ).

\section{Discussion}

This study pertains to two issues: the role of the amygdala in CTA learning and memory, and the role of protein synthesis in general, and c-Fos translation in particular, in these processes. Our results indicate that the transient expression of c-Fos in the amygdala during or immediately after training is obligatory for normal learning and memory of taste aversion.

The role of the amygdala in learning and memory, and especially in emotional learning and memory, has been well documented (for review, see Davis 1992; LeDoux 1993; McGaugh et al. 1993; Gallagher and Holland 1994; Cahill et al. 1995). Some evidence suggests that storage of recently acquired information, particularly affective memory, occurs within the amygdala itself, whereas other evidence suggests that the amygdala also plays a critical role in regulating memory storage in other brain regions (McGaugh et al. 1995). A number of studies have specifically addressed the role of the amygdala in CTA learning and memory, by use of targeted lesions. The overall conclusions are still debated, and the results appear to depend on the type of lesion (whether surgical ablation, electrolytic, cytotoxic, or metabolic lesion), its location within the amygdala, its duration (permanent vs. transient), and its timing relative to training and testing. Although some investigations revealed no significant effects of certain amygdala lesions on CTA (Kemble et al. 1979; Hatfield et al. 1992; Galaverna et al. 1993), in most studies, amygdala lesions, especially of the basolateral nucleus (Fitzgerald and Burton 1983; Simbayi et al. 1986; Yamamoto and Fujimoto 1991; Roldan and Bures 1994; Yamamoto et al. 1995), but also including the CeA (Lasiter and Glanzman 1982 1985; Roldan and Bures 1994), disrupted acquisition of CTA, its retrieval, or both. Roldan and Bures (1994) suggested that amygdaloid nuclei subserve CTA in the intact brain, but that their permanent absence can be compensated for by other brain centers.
We have recently demonstrated, using in situ hybridization, that the expression of the IEG c-fos mRNA in the CeA is markedly increased $40 \mathrm{~min}$ after the administration of $\mathrm{LiCl}$ i.p. (Lamprecht and Dudai 1995). In contrast, we found no c-fos mRNA modulation in the amygdala after sampling an unfamiliar taste alone (Lamprecht and Dudai 1995). Thus, our gene expression data indicate that the CeA is involved in processing and/or storing information about the unconditioned aversive stimulus (UCS), that is, $\mathrm{LiCl}$ i.p., in CTA training. These data do not, of course, exclude the involvement of other IEGs and other amygdaloid nuclei in representing the UCS, the conditioned stimulus (CS, the unfamiliar taste), or their association, in CTA (Roldan and Bures 1994; Yamamoto et al. 1995). Furthermore, the in situ hybridization technique yielded only correlative results and did not determine whether the expression of the IEG mRNA in the CeA is obligatory for the proper representation of the UCS in CTA training.

By locally, transiently, and specifically blocking c-Fos expression with antisense ODNs, and by locally, transiently, and generally blocking translation with anisomycin, we have been able to show that the amygdala is, indeed, obligatory for the normal acquisition of CTA memory. Our microinjection coordinates were guided by the c-fos expression data, which implicated the CeA in the representation of the UCS (Gu et al. 1993; Yamamoto et al. 1993; Lamprecht and Dudai 1995). However, a caveat is appropriate here. The sphere of diffusion of both antisense ODNs and anisomycin exceeds the borders of the CeA (see Materials and Methods and Results), and the effect of protein synthesis inhibition is, of course, not confined to c-Fos expression. Furthermore, the time course of the action of antisense ODNs and of anisomycin overlaps the time of administration of both the CS and the UCS. Clearly, anisomycin is more effective in blocking CTA memory when it overlaps the administration of the CS also, not merely the UCS. Therefore, at this stage we can conclude only that an area overlapping CeA is obligatory for CTA in the normal (i.e., not permanently lesioned) brain, but cannot delineate further the anatomical sites or the exact time windows of acquisition and consolidation during which these anatomical sites are functionally recruited. In this context, it is of interest to note that Houpt et al. (1995) recently reported induction of $\mathrm{c}$-Fos in the CeA in response to the CS in CTA testing. We found no significant effect of inhibition of c-Fos expression on CTA at

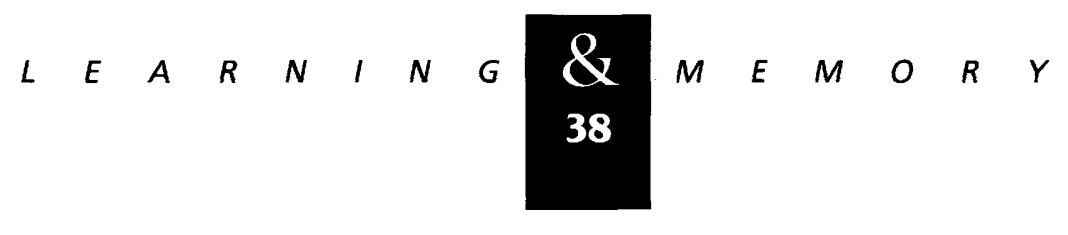


this stage. The difference may stem from either a different CTA paradigm [Houpt et al. (1995) administered the CS by infusion], or from a correlative but not obligatory role of the IEG in retrieval or in relearning during the unpaired administration of the CS in the test.

The observation that an IEG is obligatory for normal CTA learning is consonant with a prevailing molecular hypothesis of memory consolidation (Goelet et al. 1986; Dudai 1989). According to this hypothesis, the stimuli that trigger the encoding of long-term memory initiate molecular cascades that modulate IEGs, which in turn modulate the expression of late response genes, culminating in lasting alterations in the neuronal circuits that encode the stored representation. We show here that in a behaving animal, the normal expression of c-Fos, a protein that forms functional dimers with members of the jun family to produce the AP-1 transcription factors (Nakabeppu et al. 1988), is necessary for normal learning of a specific task in a specific brain region.

Our study took advantage of the fact that CTA is a single-trial learning paradigm and that an increased c-Fos expression in the CeA was found to correlate with LiCl i.p. ( $\mathrm{Gu}$ et al. 1992; Yamamoto et al. 1993; Lamprecht and Dudai 1995). This enabled us to target the IEG to the anatomical site within a circumscribed, relatively brief time window. This is especially important when using antisense ODNs. These reagents are expected to block translation by multiple mechanisms. In addition to inhibition of the interaction of the target mRNA with the ribosome and translational factors, phosphorothioate ODNs form an ODN-mRNA hybrid that is a substrate for RNase $\mathrm{H}$, which recognizes DNA-RNA hybrids and cleaves the RNA (Gao et al. 1991). Additional effects include inhibition rather than activation of RNase $H$ and inhibition of DNA polymerases (Gao et al. 1991; Helene 1991). Some of these effects are nonsequence-specific and increase with the length of the ODNs, the dose, and the duration of application (Gao 1991; Helene 1991). We have used relatively short antisense ODNs, which decrease nonspecific toxicity (Gao et al. 1991), but even under these conditions marked tissue toxicity emerges with repetitive or prolonged application (Chiasson et al. 1994). Therefore, the ability to use a single antisense ODN application in our protocol is an advantage.

Clearly, c-Fos is only one of the IEGs expected to play a role in CTA memory consolidation in the brain, in general, and in the amygdala, in particular (Lamprecht and Dudai 1995). It is plausible to assume that in each of the brain areas involved in the internal representation of the CS, UCS, and their association in CTA (Yamamoto et al. 1994), a differential and combinatorial modulation of IEGs' mRNA cellularly encodes the relevant stimuli (He and Rosenfeld 1991; Struhl 1991). It would be interesting to elucidate the role of other IEGs as well as of late-response genes in encoding CTA by use of targeted, local application of specific antisense ODNs into candidate brain areas.

\section{Acknowledgments}

We are grateful to Burton M. Slotnick for critical comments on the manuscript and for advice on the statistical analysis, and to Shoshi Hazvi for assistance in the histological analysis. This study was supported by a grant from the US-Israel Binational Science Foundation (BSF), Jerusalem.

The publication costs of this article were defrayed in part by payment of page charges. This article must therefore be hereby marked "advertisement" in accordance with 18 USC section 1734 solely to indicate this fact.

\section{References}

Bures. J., O. Buresova, and J. Krivanek. 1988. Brain and behavior: Paradigms for research on neural mechanisms. Wiley, New York, NY.

Cahill, L., R. Babinsky, H.J. Markowitch, and J.L. McGaugh. 1995. The amygdala and emotional memory. Nature 377: 295-296.

Chiasson, B.J., M.L. Hopper, P.R. Murphy, and H.A. Robertson. 1992. Antisense oligonucleotide eliminates in vivo expression of c-fos in mammalian brain. Eur. J. Pharmacol. 227: 451-453.

Chiasson, B.J., J.N. Armstrong, M.L. Hooper, P.R. Murphy, and H.A. Robertson. 1994. The application of antisense oligonucleotide technology to the brain: Some pitfalls. Cell. Mol. Neurobiol. 14: 507-520.

Cirelli, C., M. Pompeiano, and G. Tononi. 1995. In vivo antisense approaches to the role of immediate early gene expression in the brain. Regul. Pept. 59: 151-162.

Davis, M. 1992. The role of the amygdala in fear and anxiety. Ann. Rev. Neurosci. 15: 353-375.

Davis, H.P. and L.R. Squire. 1984. Protein synthesis and memory: A review. Psychol. Bull. 96: 518-559.

Dragunow, M., P. Lawlor, B. Chiasson, and H. Robertson. 1993. c-fos antisense generates apomorphine and amphetamine-induced rotation. NeuroReport 5: 305-306.




Dudai, Y. 1989. The neurobiology of memory. Concepts, findings, trends. Oxford University Press, Oxford, UK.

Edwards, D.R. and Mahadevan, L.C. 1992. Protein synthesis inhibitors differentially superinduce $\mathrm{c}$-fos and $\mathrm{c}$-jun by three distinct mechanisms: Lack of evidence for labile repressors. EMBO J. 11: 2415-2424.

Fitzgerald, R.E. and M.J. Burton. 1983. Neophobia and conditioned taste aversion deficits in the rat produced by undercutting temporal cortex. Physiol. Behav. 30: 203-206.

Galaverna, O.G., R.J. Seeley, K.C. Berridge, H.J. Grill, A.N. Epstein, and J. Schulkin. 1993. Lesions of the central nucleus of the amygdala. I: Effects of taste reactivity, taste aversion learning and sodium appetite. Behav. Brain Res. 59: 11-17.

Gallagher, M. and P.C. Holland. 1994. The amygdala complex: Multiple roles in associative learning and attention. Proc. Natl. Acad. Sci. 91: 11771-11776.

Gallo, M., G. Roldan, and J. Bures. 1992. Differential involvement of gustatory insular cortex and amygdala in the acquisition and retrieval of conditioned taste aversion in rats. Behav. Brain Res. 52: 91-97.

Gao, W.-Y., F.-S. Han, C. Storm, W. Egan, and Y.-C. Cheng. 1991. Phosphorothioate oligonucleotides are inhibitors of human DNA polymerases and RNase $\mathrm{H}$ : Implications for antisense technology. Mol. Pharmacol. 41: 223-229.

Goellet, P., V.F. Castellucci, S. Schacher, and E.R. Kandel. 1986. The long and the short of long-term memory - A molecular framework. Nature 322: 419-422.

Grollman, A.P. 1967. Mode of action of anisomycin. I. Biol. Chem. 242: 3226-3233.

Gu, Y., M.F. Gonzalez, D.Y. Chin, and J.A. Deutch. 1993. Expression of $\mathrm{c}$-fos in brain subcortical structures in response to nausant lithium chloride and osmotic pressure in rats. Neurosci. Lett. 157: 49-52.

Hatfield, T., P.W. Graham, and M. Gallagher. 1992. Taste-potentiated odor aversion learning: Role of the amygdaloid basolateral complex and central nucleus. Behav. Neurosci. 106: 286-293.

He, X. and M.G. Rosenfeld. 1991. Mechanisms of complex transcription regulation: Implication for brain development. Neuron 7: 183-196.

Helene, C. 1991. Rational design of sequence-specific oncogene inhibitors based on antisense and antigene oligonucleotides. Eur. J. Cancer 11: 1466-1471.

Heilig, M., J.A. Engel, and B. Soderpalm. 1993. c-fos antisense in the nucleus accumbens blocks the locomotor stimulant action of cocaine. Eur. J. Pharmacol.

236: 339-340.

Houpt, T.A., R.A. Berlin, and G.P. Smith. 1995. Altered induction of c-Fos in the central nucleus of the amygdala $(\mathrm{CeN})$ correlated with conditioned taste aversion expression. Soc. Neurosci. Abst. 21: 1682.

Kemble, E.D., D.R. Studelska, and M.K. Schmidt. 1979. Effects of central amygdaloid nucleus lesions on ingestion, taste reactivity, exploration and taste aversion. Physiol. Behav. 22: 789-793.

Lamprecht, R. and Y. Dudai. 1995. Differential modulation of brain immediate early genes by intraperitoneal $\mathrm{LiCl}$. NeuroReport 7: 289-293.

Landgraf, R., R. Gerstberger, A. Montkowski, J.C. Probst, C.T. Wotjak, F. Holsboer, and M. Engelmann. 1995. V1 vasopressin receptor antisense oligodeoxynucleotide into septum reduces vasopressin binding, social discrimination abilities, and anxiety-related behavior in rats. /. Neurosci. 15: $4250-4258$.

Lasiter, P.S. and D.L. Glanzman. 1982. Cortical substrates of taste aversion learning: Dorsal prepiriform (insular) lesions disrupt taste aversion learning. I. Comp. Physiol. Psychol. 96: $376-392$.

1985. Cortical substrates of taste aversion learning: Involvement of dorsolateral amygdaloid nuclei and temporal neocortex in taste aversion learning. Behav. Neurosci. 99: $257-276$.

LeDoux, J.E. 1993. Emotional memory: In search of systems and synapses. Ann. NY Acad. Sci. 702: 149-157.

McGaugh, J.L., I.B. Introni-Collison, L.F. Cahill, C. Castellano, C. Dalmaz, M.B. Parent, and C.L. Williams. 1993. Neuromodulatory systems and memory storage: Role of the amygdala. Behav. Brain Res. 58: 81-90.

McGaugh, J.L., L. Cahill, M.B. Parent, M.H. Mesches, K. Coleman-Mesches, and J.A. Salinas. 1995. Involvement of the amygdala in the regulation of memory storage. In Plasticity in the nervous system. Learning and Memory. (ed. J.L. McGaugh, F. Bermudez-Rattoni, and R.A. Prado-Alcala), pp. 17-39. Lawrence Erlbaum Associates, Hillsdale, New Jersey.

Moller, C., O. Bing, and M. Heilig. 1994. c-fos expression in the amygdala: In vivo antisense modulation and role in anxiety. Cell. Mol. Neurobiol. 14: 415-423.

Nakabeppu, Y., K. Ryder, and D. Nathans. 1988. DNA binding activities of three murine Jun proteins: Stimulation by Fos. Cell 55: 907-915.

Naor, C. and Y. Dudai. 1996. Transient impairment of cholinergic function in the rat insular cortex disrupts the encoding of taste in conditioned taste aversion. Behav. Brain. Res. (in press).

Norgren, R. 1976. Taste pathways to hypothalamus and amygdala. I. Comp. Neurol. 166: 17-30.

Paxinos, G. and C. Watson. 1986. The rat brain in

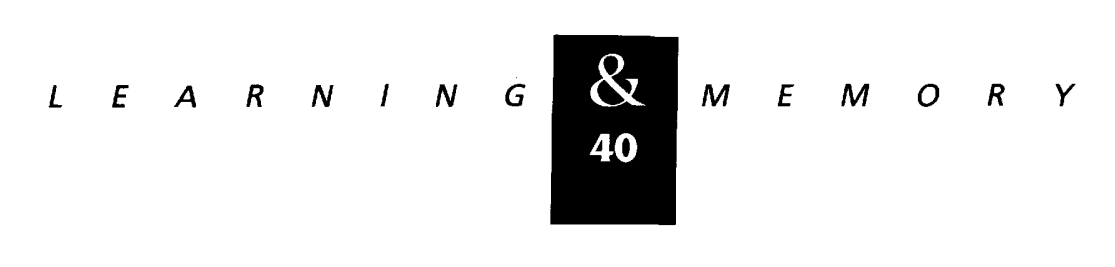


stereotaxic coordinates. 2nd ed. Academic Press, San Diego, CA.

Revusky, S. and J. Garcia. 1970. Learned associations over long delays. Psychol. Learn. Motiv. 4: 3-84.

Roldan, G. and J. Bures. 1994. Tetrodotoxin blockade of amygdala overlapping with poisoning impairs acquisition of conditioned taste aversion in rats. Behav. Brain Res.

65: 213-219.

Rosenblum, K., N. Meiri, and Y. Dudai. 1993. Taste memory: The role of protein synthesis in gustatory cortex. Behav. Neural Biol. 59: 49-56.

Rosenblum, K., R. Schul, N. Meiri, Y. Hadari, Y. Zick, and Y. Dudai. 1995. Modulation of protein tyrosine phosphorylation in rat insular cortex following conditioned taste aversion training. Proc. Natl. Acad. Sci. 92: 1157-1161.

Simbayi, L.C., R.A. Boakes, and M.J. Burton. 1986. Effects of basolateral amygdala lesions on taste aversions produced by lactose and lithium chloride in the rat. Behav. Neurosci. 100: $455-465$.

Sommer, W., B. Bjelke, D. Ganten, and K. Fuxe. 1993. Antisense oligonucleotide to $\mathrm{c}$-fos induces ipsilateral rotational behavior to $d$-amphetamine. NeuroReport 5: $277-280$

Struhl, K. 1991. Mechanisms for diversity in gene expression patterns. Neuron 7: 177-181.

Turner, B.H. and M. Herkenham. 1991. Thalamoamygdaloid projections in the rat: A test of the amygdala's role in sensory processing. I. Comp. Neurol. 313: 295-325.

van der Kooy, D., L.Y. Koda, J.F. McGinty, C.R. Gerfen, and F.E. Bloom. 1984. The organization of projections from the cortex, amygdala, and hypothalamus to the nucleus of the solitary tract in rat. J. Comp. Neurol. 224: 1-24.

Wahlestedt, C. 1994. Antisense oligonucleotide strategies in neuropharmacology. Trends Pharmacol. Sci. 15: 42-46.

Yamamoto, T. and Y. Fujimoto. 1991. Brain mechanisms of taste aversion learning in the rat. Brain Res. Bull.

27: 403-406.

Yamamoto, T., T. Shimura, N. Sako, S. Azuma, W.Z. Bai, and S. Wakisaka. 1993. c-Fos expression in rat brain after intraperitoneal injection of lithium chloride. NeuroReport 3: $1049-1052$.

Yamamoto, T., T. Shimura, N. Sako, Y. Yasoshima, and N. Sakai. 1994. Neural substrates for conditioned taste aversion in the rat. Behav. Brain Res. 65: 123-137.

Yamamoto, T., Y. Fujimoto, T. Shimura, and N. Sakai. 1995. Conditioned taste aversion in rats with excitotoxic brain lesions. Neurosci. Res. 22: 31-49.

Received February 6, 1996; accepted in revised form June 28, 1996.

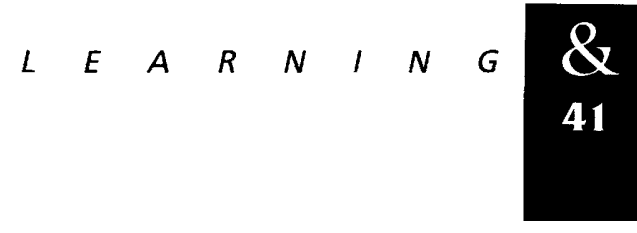






\section{Transient expression of c-Fos in rat amygdala during training is required for encoding conditioned taste aversion memory.}

R Lamprecht and $Y$ Dudai

Learn. Mem. 1996, 3:

Access the most recent version at doi:10.1101//m.3.1.31

References This article cites 45 articles, 4 of which can be accessed free at: http://learnmem.cshlp.org/content/3/1/31.full.html\#ref-list-1

License

Email Alerting Receive free email alerts when new articles cite this article - sign up in the box at the Service top right corner of the article or click here. 